\title{
A Case Report and Review of Hyperprolactinemia that is not Prolactinoma
}

\author{
Fateme Salehi, Francesca Doglietto, Rowena Ridout, Fred Gentili, Sylvia Asa, Gelareh Zadeh
}

Can. J. Neurol. Sci. 2011; 38: 652-655

Hyperprolactinemia (hyper-PRL) can be due to a wide range of etiologies, including pregnancy, primary hypothyroidism, polycystic ovary syndrome and various drugs. Once other possible causes are ruled out and when associated with a tumor in the sellar region, it is important to differentiate hyper-PRL due to the so called "stalk effect" tumor secretion: the importance of this distinction lies in the fact that prolactinomas usually respond to medical therapy, while other tumors that cause the "stalk effect" commonly require surgical intervention due to mass effect and compression of the optic chiasm. "Stalk effect" refers to compression of the infundibulum that attenuates prolactin inhibition by hypothalamic dopamine. Various reports discuss the cut-off value that distinguishes hyper-PRL produced by the tumor from the one caused by "stalk effect'; these are observational studies, inferring the cut-off values from the analysis of prolactinomas and other sellar tumors causing hyper-PRL. ${ }^{2}$ Prolactin (PRL) values of more than $250 \mathrm{ng} / \mathrm{ml}$ (normal values:1.2-29.9 ng/ml) are usually considered diagnostic of a prolactinoma ${ }^{3}$; recently some authors have suggested lowering these cut-off values. ${ }^{2}$
Although these studies certainly have a practical value, as they simplify the differential diagnosis between prolactinoma and pseudoprolactinoma, they also present some limitations. We present a case that reflects such limitations and highlights the importance of recognizing a case of a pseudoprolactinoma, with the histopathological diagnosis of a nonfunctioning adenoma associated with high prolactin values.

\section{Case Description}

A 42-year-old woman presented with a 17-month history of amenorrhea, which had been preceded by irregular menses. She denied having galactorhea. Her initial assessment and management was carried out by her local physician prior to referral to our institution. In September 2006 a complete endocrinological work up was carried out as part of her investigations and elevated prolactin levels of 277 and $369 \mathrm{ng} / \mathrm{ml}$ were documented. In March 2007 repeat prolactin was $293 \mathrm{ng} / \mathrm{ml}$ (normal range: 1.2-29.9 ng/ml). A brain magnetic resonance imaging (MRI) documented a sellar and suprasellar mass (Figure

Table: Endocrinological assessment before and after medical therapy and surgery

\begin{tabular}{|c|c|c|c|c|c|c|c|c|c|c|c|}
\hline Timing of exams & PRL & hGH & IGF-1 & $\begin{array}{c}\text { A.M. } \\
\text { Cortisol }\end{array}$ & ACTH & TSH & fT3 & fT4 & FSH & LH & Estradiol \\
\hline Before medical therapy & 500.5 & $<0.3$ & - & 348 & 3.7 & 1.49 & 3.9 & 11.0 & 8.5 & 1.2 & 1563 \\
\hline After medical therapy & $<1.0$ & $<0.3$ & 38 & 238 & 6.2 & 0.98 & 3.4 & 10.0 & 10.0 & 2.9 & - \\
\hline After surgery (3-6 months) & 3.6 & $<0.3$ & - & 320 & 7.8 & 0.20 & 3.5 & 9.5 & 8.0 & 2.5 & - \\
\hline
\end{tabular}

PRL:prolactin; hGH: human growth hormone; IGF-1: insulin-like growth factor-1; ACTH: adrenocorticotropic hormone; TSH: thyroid stimulating hormone; fT3: free liothyronine; fT4: free levothyroxine; FSH: follicle stimulating hormone; LH: luteinizing hormone. Normal values: PRL: 1.2$29.9 \mu \mathrm{g} / \mathrm{L}$; GH: <=0.9; IGF-1178-295 $\mu \mathrm{g} / \mathrm{L}$; Cortisol138-690 nmol/L; ACTH: <=21.9 pmol/L; TSH: 0.35-4.94 mIU/L; fT3: 2.6-5.7 pmol/L; fT4: 919 pmol/L; FSH: 1.1-151 IU/L; LH: 0.9-74.2 IU/L; Estradiol: 0-1462 pmol/L.

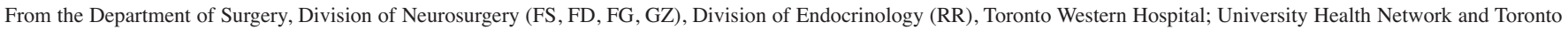
Medical Laboratories (SA), University of Toronto, Toronto, Ontario, Canada.

Received October 25, 2010. Final Revisions Submitted January 27, 2010.

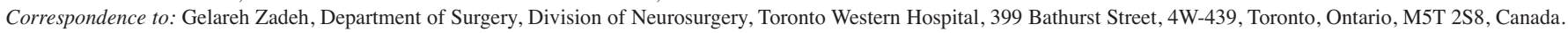



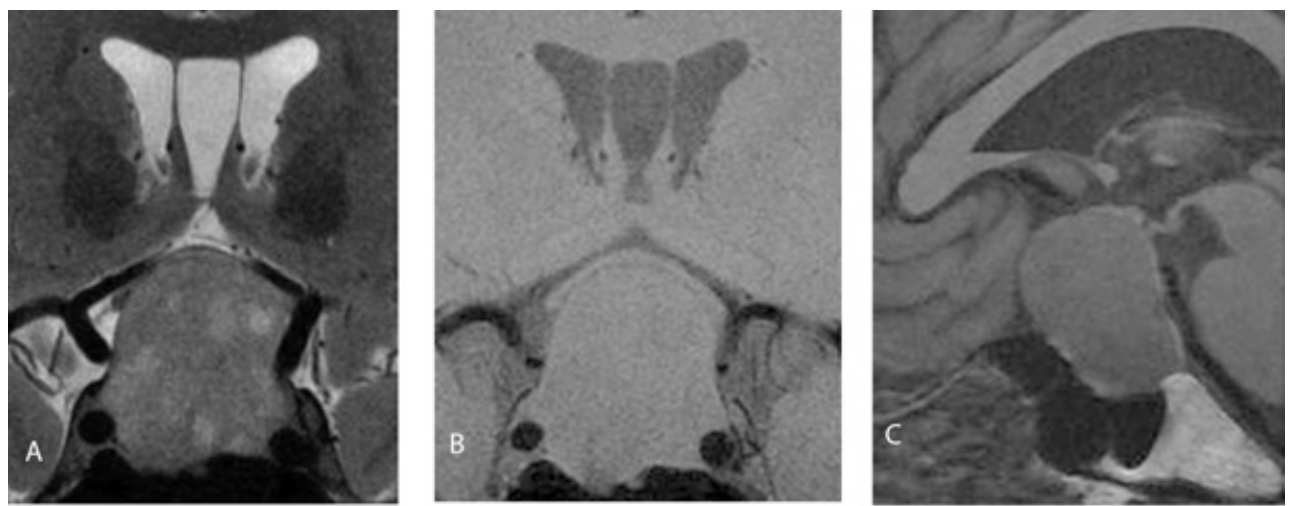

Figure 1: Pre-operative MRI. A) Coronal T2-weighted (w), B) T1-w coronal and $C$ ) sagittal images after contrast administration documenting a space occupying lesion in the sellar area, extending in the suprasellar space with compression of the chiasm
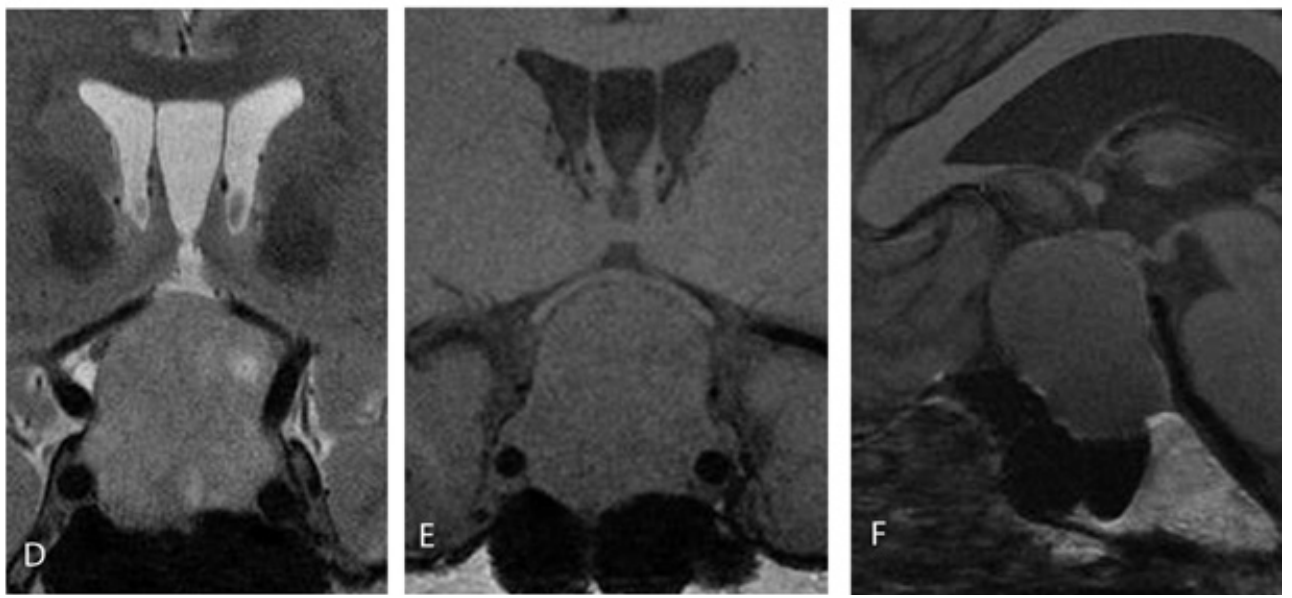
(maximum diameters: $2.8 \times 2.8 \times 3.8$ $\mathrm{cm})$; D) Coronal T2-w and E) $\mathrm{Tl}-\mathrm{w}$ coronal and $F$ ) sagittal images after contrast administration documenting no significant change in the dimensions of the lesion after further 4 months of medical therapy. T2-w images always show a heterogeneous signal, compatible with areas of a partially cystic adenoma.

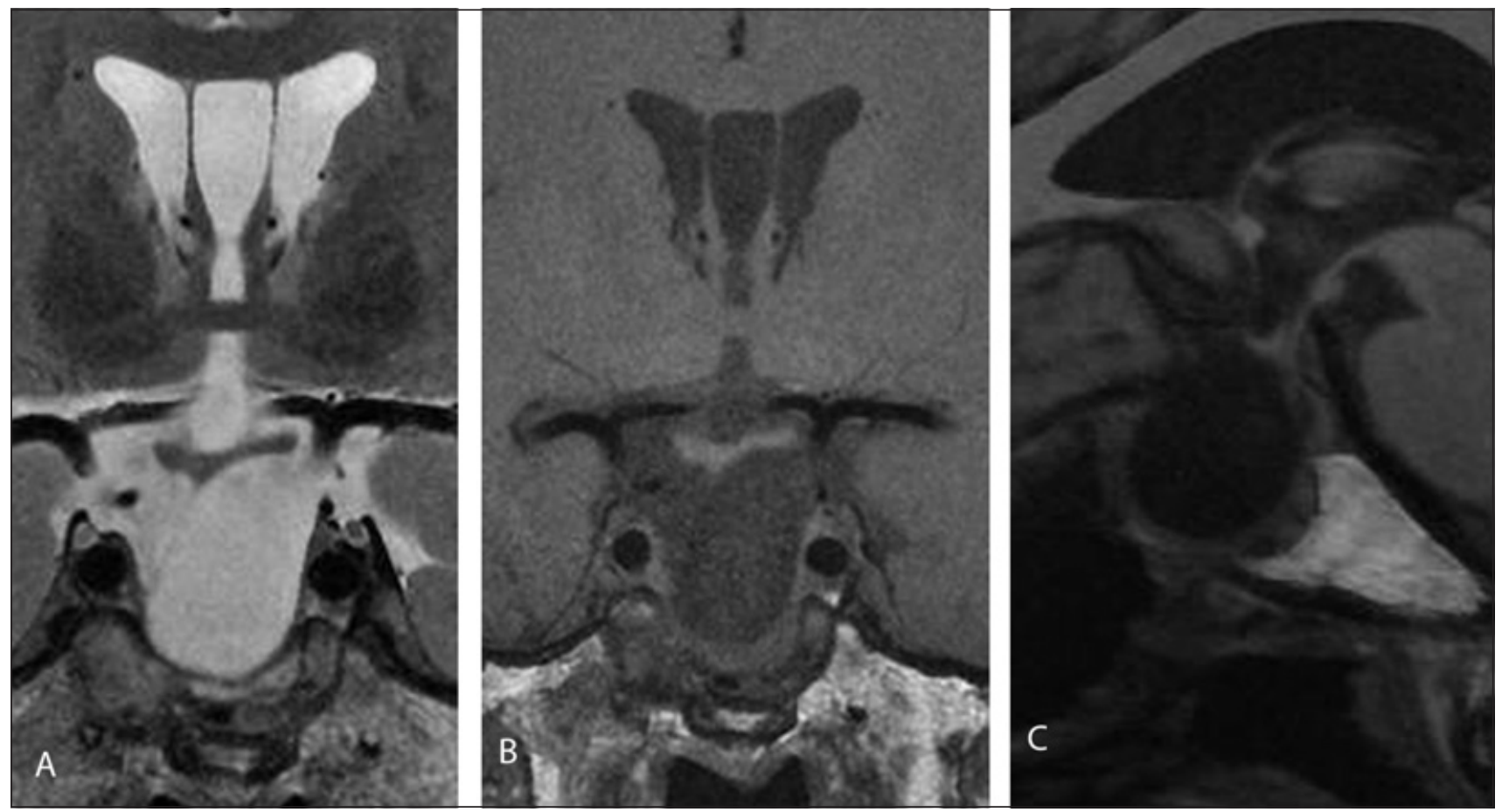

Figure 2: Post-operative MRI: T1-weighted MR images, six months after surgery, documenting complete excision of the lesion with decompression of the optic apparatus and preservation of the pituitary gland (A-B: coronal sections; $C$ : sagittal image). 
1A-C) and based on these findings the diagnosis of pituitary macroadenoma was made and initially dopamine agonist therapy was offered, but the patient refused it for personal reasons. At this point the patient was referred to our multidisciplinary pituitary clinic at Toronto Western Hospital. She remained amenhorroic and had complaints of visual blurring. Her past medical history was significant for glaucoma and a tubal ligation performed ten years previously. She had six children in good health. Her only medication was travoprost, for the treatment of glaucoma. Repeat endocrinological work up demonstrated a prolactin level of $500.5 \mathrm{ng} / \mathrm{Ml}$ (normal values: 1.2-29.9 ng/ml). Baseline pituitary hormonal assessment documented initial signs of hypopituitarism, with a deficit of the human growth hormone (hGH) axis (Table 1). Triple bolus test documented no hGH, PRL or follicle stimulating hormone (FSH) response and borderline values for cortisol, thyroid stimulating hormone (TSH) and luteinizing hormone $(\mathrm{LH})$ response. A triple bolus test is a dynamic test to assess anterior pituitary function, using either insulin, gonadotropin releasing hormone or thyrotropin releasing hormone to do provocative testing of the pituitary gland. This allows assessment in production of cortisol and growth hormone, prolactin and TSH respectively.

Visual acuity and cranial nerve exam demonstrated a decrease in visual acuity with $10 / 15$ in the left and 10/20 on the right eye. A formal visual field exam documented a bilateral upper temporal quandrantanopia and partial inferior temporal quadrantanopia. The patient was counseled to have treatment, with the indications for therapy being high-lighted to her. As the prolactin levels were significantly higher than the levels usually considered to reflect the "stalk effect", especially after repeated measurements of prolactin levels and confirmation of laboratory values, a working diagnosis of a macroprolactinoma was made and the patient was offered both medical therapy and surgery: she consented to medical therapy and was treated with cabergoline $(0.5 \mathrm{mg} /$ week $)$ with normalization of the prolactin levels at one month follow-up $(<1 \mathrm{ng} / \mathrm{ml})$. Three months later a new MRI examination was reported to document a slight reduction in size of the tumor. The patient denied any worsening in her vision and visual field testing exhibited no change. Her symptoms did not resolve with medical therapy and she continued to be amenorrhic. Further strict follow-up was planned as the reduction in size of the lesion was not considered satisfactory. A three month follow-up MRI did not document any significant change in the lesion dimensions (Figure 1D-F) despite continued medical therapy and $<1 \mathrm{ng} / \mathrm{ml}$ documented prolactin level. After a detailed discussion of the risks and benefits of surgery, with primary goals of decompressing the optic chiasm, the patient consented to proceed with elective transsphenoidal surgery. The patient underwent an endoscopic transsphenoidal tumor resection, with gross total removal of the tumor as confirmed by post-operative MRI (Figure 2).

The histological diagnosis documented a gonadotroph adenoma (Figure 3) with no evidence of prolactin positive staining cells. Post-operatively the patient experienced diabetes insipidus (DI), which resolved at six months follow-up. The patient showed evidence of secondary hypothyroidism with a TSH that was inappropriately normal in the setting of a lownormal T4. She reported marked improvement of her vision and formal visual field documented improvement in her fields. Postoperatively, the patient returned to normal menstrual cycles.

\section{Case Discussion}

Hyperprolactinemia is a relatively common cause of amenorrhea and the differential diagnosis can be extremely challenging if the prolactin levels are relatively low. Hyperprolactinemia associated with a sellar mass is usually the consequence of prolactin secretion by a pituitary adenoma or is due to the so-called "stalk effect". ${ }^{1}$ Many reports stress the

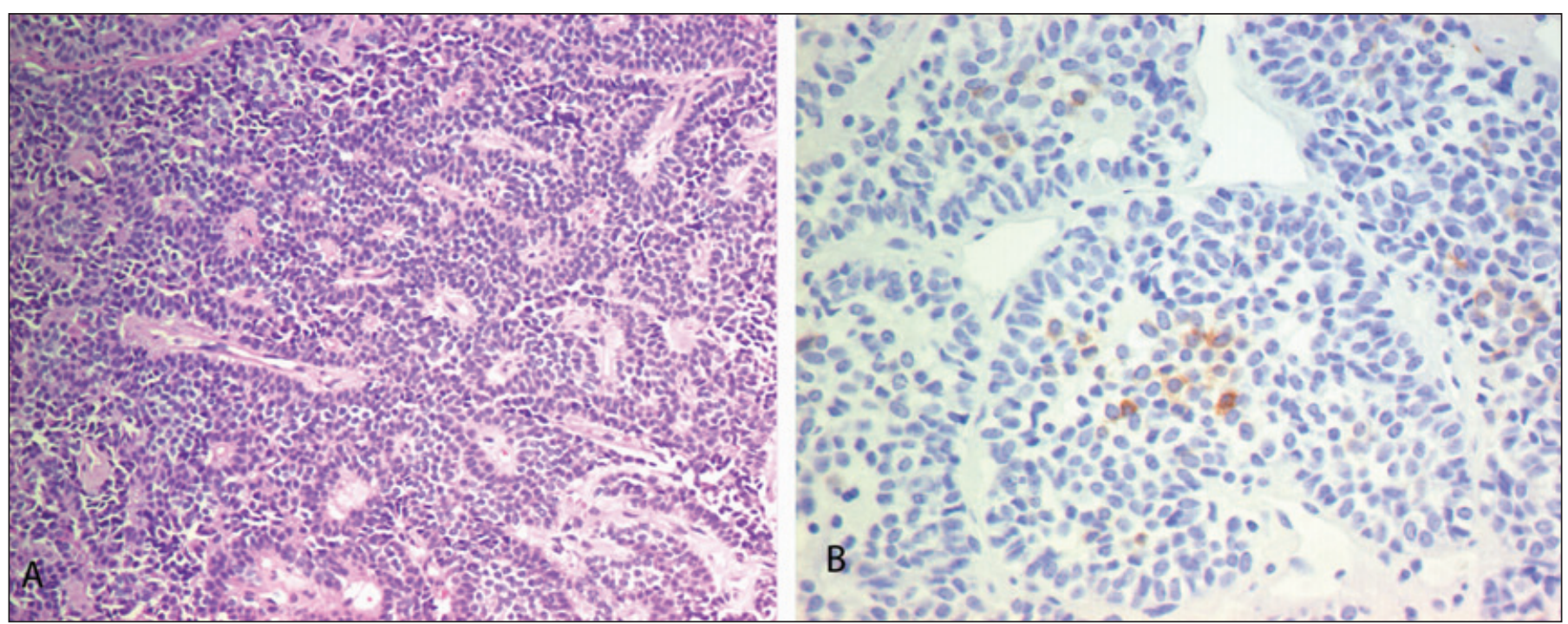

Figure 3: Histological microphotograph of the gonadotroph pituitary adenoma. A) A hematoxylin and esosin (H\&E) stain of the tumor cells demonstrating typical pseudorosette pattern associated with gonadotroph adenoma. B) Tumor cells exhibiting sparse cytoplasmic immunoreactivity for follicle stimulating hormone ( FSH). 
possibility of falsely low prolactinemia, as a consequence of the "hook effect", where extremely high serum concentrations of an antigen may paradoxically produce a lower response than expected and, on dilutions, produce a higher assay response. ${ }^{2}$ Many clinical reports and book chapters report prolactin values that should not be associated with diagnostic uncertainty, ranging from $400 \mathrm{ng} / \mathrm{ml}^{8}$ down to $100 \mathrm{ng} / \mathrm{ml}$ (or $2000 \mathrm{mU} / \mathrm{l}$ ). ${ }^{2}$ Although extremely helpful in the day to day practice, as they simplify the differential diagnosis of pituitary lesions associated with hyperprolactinemia, these cut-off values are based on induction. Moreover, some of these papers present an initial bias, as they $a$ priori exclude tumors associated with prolactin levels higher than $8000 \mathrm{MUI} / \mathrm{l}$ (equivalent to approximately $400 \mathrm{ng} / \mathrm{ml}$ ), assuming that these tumors are prolactinomas. ${ }^{2}$

Though rare, histologically confirmed non-prolactin producing sellar tumors associated with prolactin levels up to $662 \mathrm{ng} / \mathrm{ml}$ have been previously described ${ }^{1,4}$. Our case is another example of hyper-PRL, which was not associated with a prolactinoma and could be attributed to "stalk effect" or perhaps another, as yet, unidentified etiology. In the case of our patient other etiologies of elevated prolactin were ruled out. Other explanations to be considered include: 1) Existence of a double adenoma with both gonadotroph and PRL-secreting components. This association has only been described in an autopsy series of incidental pituitary adenomas and a recent case report of a double gonadotroph/PRL pituitary adenoma. ${ }^{5}$ Although the extremely small reduction in tumor dimensions is most likely due to an inter-examination variation, the hypothesis of a small prolactinoma responding to medical therapy could also explain the variation. 2) Macroprolactin, a high-weight molecular form of prolactin, which is believed to have decreased bioactivity, ${ }^{2}$ has been described as a possible cause of falsely elevated prolactin levels. However, our instituition laboratory routinely performs specific assays to ensure that macro-prolactenimia is ruled out.

In general, serum prolactin levels parallel tumor size fairly closely. In this case the PRL values, although high, were not in the typical range of a prolactin-secreting macroadenoma, usually in the thousands rather than hundreds $(\mathrm{ng} / \mathrm{ml})$ range. Although it is justified to offer a trial of dopamine agonists to patients who have high prolactin levels and a pituitary macroadenoma, all physicians should be aware of the necessity of close clinical surveillance including serial MRI to document response to the medical therapy and rule out the occasional, rare case of pseudoprolactinoma presenting with high PRL values.

\section{Conclusions}

This case highlights the rare instance of a nonfunctioning adenoma presenting with prolactin levels higher than the classic cut-off for the "stalk effect". This rare situation stresses the importance of a close follow-up of patients undergoing medical therapy in suspected prolactinomas and the necessity of a neurosurgical evaluation in cases of lack of response to medical therapy.

\section{Contributions}

FS and FD contributed equally to this manuscript.

\section{REFERENCES}

1. Smith MV, Laws ER,Jr. Magnetic resonance imaging measurements of pituitary stalk compression and deviation in patients with nonprolactin-secreting intrasellar and parasellar tumors: Lack of correlation with serum prolactin levels. Neurosurgery. 1994;34 (5):834-9.

2. Karavitaki N, Thanabalasingham G, Shore HC, et al. Do the limits of serum prolactin in disconnection hyperprolactinaemia need re-definition? A study of 226 patients with histologically verified non-functioning pituitary macroadenoma. Clin Endocrinol (Oxf). 2006;65(4):524-9.

3. Abraham P, Bevan JS. Prolactinoma. In: Powell MP, Lightman SL, Laws ERJ, editors. Management of Pituitary Tumors: The Clinician's Practical Guide. Totowa, New Jersey: Humana Press; 2003. p. 21-42.

4. Albuquerque FC, Hinton DR, Weiss MH. Excessively high prolactin level in a patient with a nonprolactin-secreting adenoma. case report. J Neurosurg. 1998;89(6):1043-6.

5. Coire CI, Smyth HS, Rosso D, Horvath E, Kovacs K. A double pituitary adenoma presenting as a prolactin-secreting tumor with partial response to medical therapy. case report. Endocr Pathol. 2010;21(2):135-8. 